\section{Comparison of intraocular pressure measured by Pascal dynamic contour tonometry and Goldmann applanation tonometry}

JYF Ku' ${ }^{1}$, HV Danesh-Meyer ${ }^{1}$, JP Craig ${ }^{1}$,

JYF Ku', HV Danesh-Meyer ${ }^{1}$, JP
GD Gamble ${ }^{2}$ and CNJ McGhee

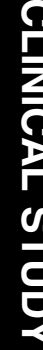

\begin{abstract}
Aims To compare the intraocular pressure (IOP) measurements obtained using the Pascal dynamic contour tonometer (PDCT) with the standard Goldmann applanation tonometer (GAT) and to correlate these with central corneal thickness (CCT) in patients with normal corneas.
\end{abstract}

Methods A prospective, masked, comparative case series of $\mathbf{1 1 6}$ eyes from patients attending a glaucoma clinic. IOP was measured with PDCT by one examiner and with GAT by a masked, independent examiner. A mean of six CCT readings was used for analysis.

Results IOP measured by the two instruments correlated significantly $(r=0.77$; $P<0.0001)$. IOP measured by GAT correlated strongly with CCT $(r=0.37, P=0.0001)$ whereas the relationship between IOP measured by PDCT and CCT approached significance $(r=0.17, P=0.073)$. The differences between GAT and PDCT measured IOP also correlated strongly with CCT ( $r=0.37, P<0.0001)$. The $95 \%$ limits of agreement between GAT and PDCT were $\pm 4.2 \mathrm{mmHg}$. Dividing the eyes into three groups on the basis of CCT, demonstrated those in the thickest tertile showed a poorer agreement between instruments and the GAT measured significantly higher IOP in this group $(P=0.003)$ while the PDCT showed no significant differences with different CCTs $(P=0.37)$.

Conclusion Demonstration of the relative independence of PDCT IOP measurements from CCT supports a potential clinical role for this instrument, particularly for subjects with CCT outside the normal range.

Eye (2006) 20, 191-198. doi:10.1038/sj.eye.6701849; published online 1 April 2005

Keywords: tonometry; intraocular pressure; corneal thickness

\section{Introduction}

Goldmann applanation tonometry (GAT) is the current gold standard for measurement of intraocular pressure (IOP). However, one limitation is that GAT measured IOP is influenced by central corneal thickness (CCT) ${ }^{1}$ since the calibration is based on a 'normal' corneal thickness of $500 \mu \mathrm{m}$ with an undisturbed corneal architecture. ${ }^{2}$ However, 'normal' CCT has been documented from 427 to $670 \mu \mathrm{m}^{3-7}$ and manometric studies reveal that applanation tonometry is most accurate when the CCT is $520 \mu \mathrm{m}$ with underestimation or overestimation in thinner or thicker corneas, respectively. ${ }^{8,9}$ This relationship between CCT and IOP has clinical implications, in particular, in the diagnosis of ocular hypertension (OHT). Indeed, several studies have documented thicker CCT in OHT subjects and suggested that some are misclassified due to thicker corneas producing an artificially raised IOP. ${ }^{6,10,11}$ Conversely, subjects with thicker corneas have been shown to have a lower rate of progression to glaucomatous damage. ${ }^{12,13}$ To ensure that only appropriate OHT subjects are commenced on long-term therapy, there is a need for accurate tonometry that is independent of CCT.
${ }^{1}$ Department of Ophthalmology, Faculty of Medical and Health Sciences, University of Auckland, Auckland, New Zealand

${ }^{2}$ Department of Medicine, Faculty of Medical and Health Sciences, University of Auckland, Auckland, New Zealand

Correspondence: $\mathrm{CNJ}$ McGhee, Department of Ophthalmology, Private Bag 92019, The University of Auckland, Auckland, New Zealand

Tel: + 6493737599 ext 86712; Fax: +649367 7173 E-mail: c.mcghee@ auckland.ac.nz

Received: 19 September 2004

Accepted: 5 January 2005 Published online: 1 April 2005 
The relative undermeasurement of IOP by GAT in thinned and relatively flattened corneas following myopic photorefractive keratectomy (PRK) was reported a decade ago ${ }^{14}$ and this phenomenon has become increasingly important with the widespread popularity of laser in situ keratomileusis (LASIK). In general, studies report a small decrease in measured IOP following corneal refractive surgery ${ }^{15-17}$ and this may delay early detection and treatment of glaucoma leading to irreversible visual loss. ${ }^{18,19}$ This is of particular concern as refractive surgery is most frequently performed in myopic subjects who have a two- to four-fold increased risk of glaucoma. ${ }^{20}$

The clinical importance of CCT in the management of $\mathrm{OHT}$ and postrefractive surgery eyes has led to increasing interest in developing a clinically applicable tonometer, unaffected by corneal thickness. The Pascal dynamic contour tonometer (PDCT) (Swiss

Microtechnology AG, Switzerland) is a new slit-lampmounted, nonapplanation, digital, contact tonometer that claims to be independent of corneal structural properties. It is based on direct measurement of the IOP by a sensor integrated into the centre of the tip. The concave surface of the tip closely matches the corneal contour when the pressures on both sides of the cornea are equal and thus induces the least possible amount of geometric deformation. A recent study comparing dynamic contour tonometry (DCT) and GAT, before and after myopic LASIK correction noted no significant change in DCTmeasured IOP while the GAT-measured IOP decreased significantly. ${ }^{21}$ The purpose of the current study was to compare masked IOP measurements obtained by PDCT and GAT and to correlate them with CCT, in a group of patients with normal corneas.

\section{Materials and methods}

In a prospective, single centre study, 116 consecutive patients were recruited from a subspeciality glaucoma clinic. Patients with a history of corneal disease, refractive surgery, and ocular trauma were excluded. To minimize systematic bias, only the right eye of each patient was included in the study. The research adhered to the tenets of the Declaration of Helsinki and was approved by the Auckland Ethics Committee. After detailed explanation informed consent was obtained from each patient prior to examination. One drop of Ophthetic ${ }^{\circledR}$ (proxymetacaine hydrochloride $5 \mathrm{mg} / \mathrm{ml}$, Allergan Australia Pty Ltd) was instilled immediately prior to IOP measurement. In $50 \%$ of subjects, GAT (Haag-Streit, Switzerland) was performed prior to PDCT and the sequence was reversed in the other half. The IOP was measured by one of two persons who did not perform the PDCT measure and were masked to the
PDCT result. Likewise, when the PDCT was performed second, the examiner was masked to the GAT result. All readings were performed within a 20 -min period for the same eye. In keeping with normal clinical practice, a single IOP measurement was performed with each instrument. This was repeated if difficulties were encountered or if the quality score for the PDCT was greater than 3 (see below). All subjects were asked to state their preference for either of the tonometers and the reason(s) for any preference.

The PDCT consists of a sensor tip with a $10.5 \mathrm{~mm}$ radius of curvature, a concave surface contour and a miniaturized pressure sensor ( $1.2 \mathrm{~mm}$ diameter) integrated into the centre of the contact surface. It allows simultaneous recordings of IOP and ocular pulse amplitude (OPA). Three conditions must be met for the measurements to be independent of corneal properties. Firstly, the radius of curvature of the cornea must be less than that of the tonometer tip, hence not exceeding $10.4 \mathrm{~mm} .{ }^{22}$ Secondly, the diameter of the contact area between the sensor tip and cornea must be larger than the diameter of the pressure sensor area, limiting the corneal radius of curvature to $5 \mathrm{~mm}$ or greater. ${ }^{22}$ Lastly, the corneal thickness must be between 300 and $700 \mu \mathrm{m}^{22}$ For each subject, a new disposable sensor tip cover is applied, which requires the use of a sterile air jet. If trapped air is observed under the tip cover, the tip cover is removed and reapplied. Correct positioning is indicated by an audible signal with a pitch proportional to the IOP. The pressure signal is detected over $4-5 \mathrm{~s}$ and the IOP is displayed in conjunction with the OPA and the quality score $(Q)$. Once displayed, the measurements cannot be altered thus minimizing operator bias. The $Q$ ranges from 1 to 6 , where 1 denotes optimum quality and 4 or higher denotes an increasingly unreliable result.

CCT was measured immediately after IOP measurements by ultrasound pachymetry (Cilco Sonometrics, USA). The mean of six CCT readings was recorded for analysis.

\section{Statistical analysis}

The sample size was pragmatically determined to provide adequate power $(80 \%$ at the $5 \%$ significance level) to detect correlations of at least 0.25 between each method and is similar, in terms of the number of eyes, to other published papers.

The GAT results were plotted against the PDCT results with a least-squares regression line fitted to test the hypothesis that the intercept of that line was $(0,0)$ and the slope $=1$. The method of Bland and Altman was used to calculate the limits of agreement. ${ }^{23}$ The plots were inspected for heteroscedasticity and a least-squares regression line fitted to explore the consistency of 
agreement between the methods over the range of intraocular pressures.

To examine the role of CCT in IOP measurement by each method, the difference between them was plotted against the CCT. The study sample was ranked by CCT and then stratified into tertiles (Table 1),

thin $=458.54 \pm 21.18 \mu \mathrm{m}(400-481 \mu \mathrm{m})$, intermediate $=499.63 \pm 8.95 \mu \mathrm{m}(485-518 \mu \mathrm{m})$, and thick $=551.25 \pm 27.34 \mu \mathrm{m}(519-625 \mu \mathrm{m})$. Individual Bland and Altman plots were constructed for each tertile.

The hypothesis that agreement between the methods differed at different corneal thicknesses and that this was related to the diagnosis of glaucoma was tested using a two-way analysis of variance (ANOVA). Significant main and interaction effects were further explored using the method of Tukey.

All tests were performed using the analysis program SAS (SAS Institute Inc, V 8.12), were two-tailed, and a 5\% significance level was maintained throughout.

\section{Results}

Of 116 consecutive patients recruited, 10 subjects were excluded due to previous refractive surgery $(n=2)$, axial corneal scarring $(n=1)$, poor cooperation $(n=1)$, and inability to obtain CCT $(n=6)$. Of the remaining 106 patients (41 males, 65 females), the mean age was $63.5 \pm 15.6$ (range 20-91) years. The diagnostic subgroups included: no glaucoma $(n=22)$, glaucoma suspect $(n=25)$, OHT $(n=5)$, normal tension glaucoma $(n=12)$, primary open angle glaucoma (POAG) $(n=39)$, and chronic angle closure glaucoma $(n=3)$ (Table 2$)$. All

Table 1 Central corneal thickness in tertiles

\begin{tabular}{lccc}
\hline & Number $(\mathrm{n})$ & Mean $\pm S D(\mu \mathrm{m})$ & Range $(\mu \mathrm{m})$ \\
\hline Total & 106 & $503.60 \pm 43.37$ & $400-625$ \\
Thin & 35 & $458.54 \pm 21.18$ & $400-481$ \\
Intermediate & 35 & $499.63 \pm 8.95$ & $485-518$ \\
Thick & 36 & $551.25 \pm 27.34$ & $519-625$ \\
\hline
\end{tabular}

Table 2 Demographics and diagnostic groups

\begin{tabular}{lcr}
\hline & Number $(\mathrm{n})$ & $(\%)$ \\
\hline Male & 41 & 39.0 \\
Female & 65 & 61.0 \\
No glaucoma & 22 & 20.8 \\
Glaucoma suspect & 25 & 23.6 \\
Ocular hypertension & 5 & 4.7 \\
Normal tension glaucoma & 12 & 11.0 \\
Primary open-angle glaucoma & 39 & 37.0 \\
Chronic angle closure glaucoma & 3 & 3.0 \\
\hline
\end{tabular}

patients with glaucoma were treated with monotherapy or combined topical therapy. GAT-measured IOP was $15.9 \pm 3.8 \mathrm{mmHg}($ mean $\pm \mathrm{SD})$ and by PDCT was $17.8 \pm 3.4 \mathrm{mmHg}$. The PDCT measurements were on average $2.0 \pm 2.1 \mathrm{mmHg}$ higher than GAT values $(P<0.0001)$. There was a significant linear relationship between PDCT and GAT $(r=0.77 ; P<0.0001)$.

The limits of agreement between the two measurements were -6.2 and $2.3 \mathrm{mmHg}$, that is, the measurements obtained by the two techniques were within $\pm 4.2 \mathrm{mmHg}$ of each other in $95 \%$ of our sample (Figure 1). A least-squares regression line was fitted and inspection of the plot demonstrated little heteroscedasticity $(P=0.42)$.

The mean CCT was $503.60 \pm 43.37 \mu \mathrm{m}$ (range 400 $625 \mu \mathrm{m})$. The different diagnostic subgroups did not show statistically significant differences in mean CCT (all $P>0.11$ ). IOP measured by GAT correlated strongly with CCT $(r=0.37, P=0.0001)$ whereas the relationship between IOP measured by PDCT and CCT approached significance $(r=0.17, P=0.073)$ (Figure 2$)$. The differences between GAT and PDCT measured IOP also correlated strongly with CCT (Figure 3$)(r=0.37$, $P<0.0001)$. Linear regression analysis showed a mean change of $0.28 \mathrm{mmHg}$ in IOP measured by GAT per $10 \mu \mathrm{m}$ change in CCT. In contrast, IOP measured by PDCT showed a change of $0.10 \mathrm{mmHg}$ per $10 \mu \mathrm{m}$ change in CCT.

The difference in IOP measured by the PDCT or GAT tonometer was not related to glaucoma status $(P=0.31)$ nor was an interaction identified between glaucoma status and the tertiles of CCT $(P=0.086)$. However, the IOP measured by the two instruments did differ between the tertiles of CCT $(P=0.0005)$. Post hoc exploration of this effect using the method of Tukey showed that those

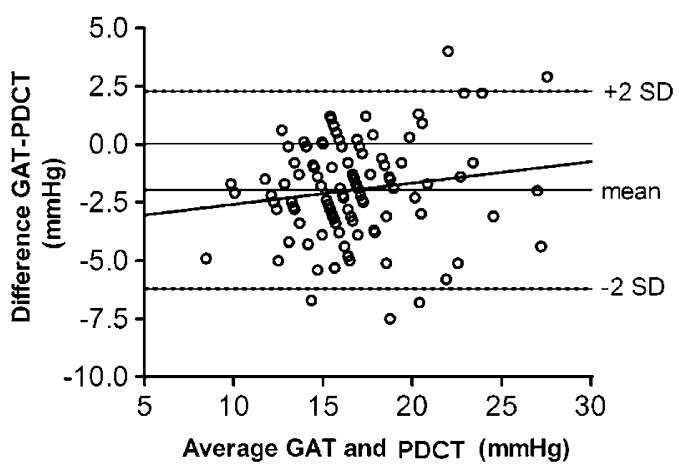

Figure 1 Difference between GAT and PDCT IOP measurements, plotted against the mean of the two measurements, for each subject together with mean difference (solid line) and 95\% confidence limits (dashed lines). The measurements obtained by these two techniques were found to be within $\pm 4.2 \mathrm{mmHg}$ of each other in $95 \%$ of cases. 


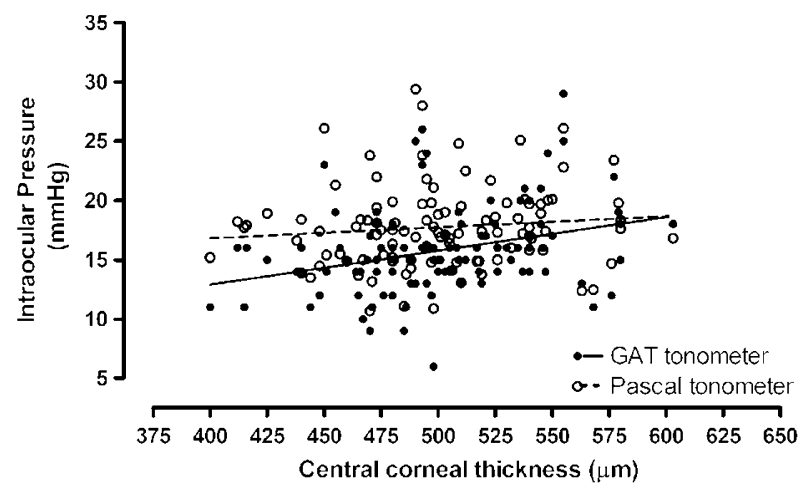

Figure 2 IOP measurements plotted against the CCT. The slope of GAT IOP measurements vs CCT regression line is 0.028 (95\% CI 0.011-0.045), while the slope of PDCT IOP measurements vs CCT regression line is -0.0093 (95\% CI -0.0069 to 0.026 ). The correlation between the PDCT and CCT approached significance $(r=0.17, P=0.07)$, in contrast, the GAT showed greater correlation with CCT $(r=0.37, P=0.0001)$.

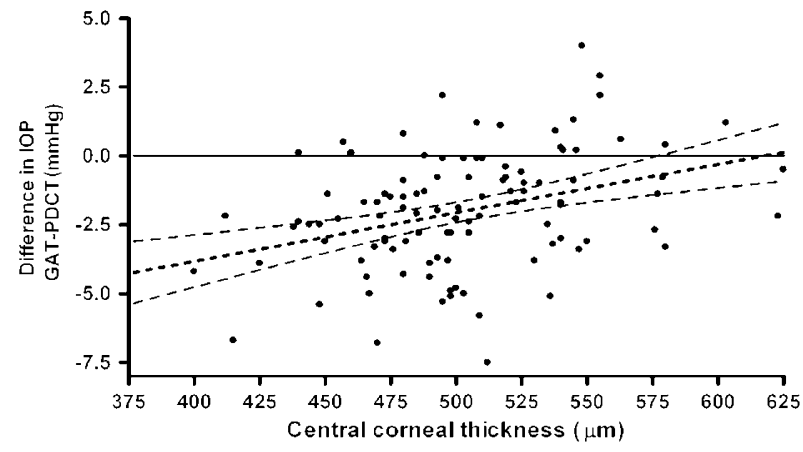

Figure 3 Difference between GAT and PDCT IOP measurements, plotted against the CCT. The slope of the regression line is 0.018 (95\% CI $0.0093-0.026)$. It shows a significant correlation $(r=0.37, P<0.0001)$ at a level similar to the correlation between GAT and CCT.

in the thickest tertile of CCT showed poorer agreement between the two tonometers than those in the intermediate or thinnest tertiles of CCT. It also demonstrated that GAT produced significantly higher IOP measurements in subjects with thicker corneas $(P=0.003)$ while IOP measured using the PDCT showed no statistically significant differences with different corneal thicknesses $(P=0.37)$. Individual Bland and Altman plots were assessed for each tertile of corneal thickness (Figure $4 \mathrm{a}-\mathrm{c}$ ). At IOP $>20 \mathrm{mmHg}$, GAT measured consistently higher IOP than PDCT in those in the highest tertile for CCT (Figure 4c). The mean OPA was $2.8 \pm 1.2 \mathrm{mmHg}$, which was not significantly different in those with or without glaucoma $(P=0.13)$. a
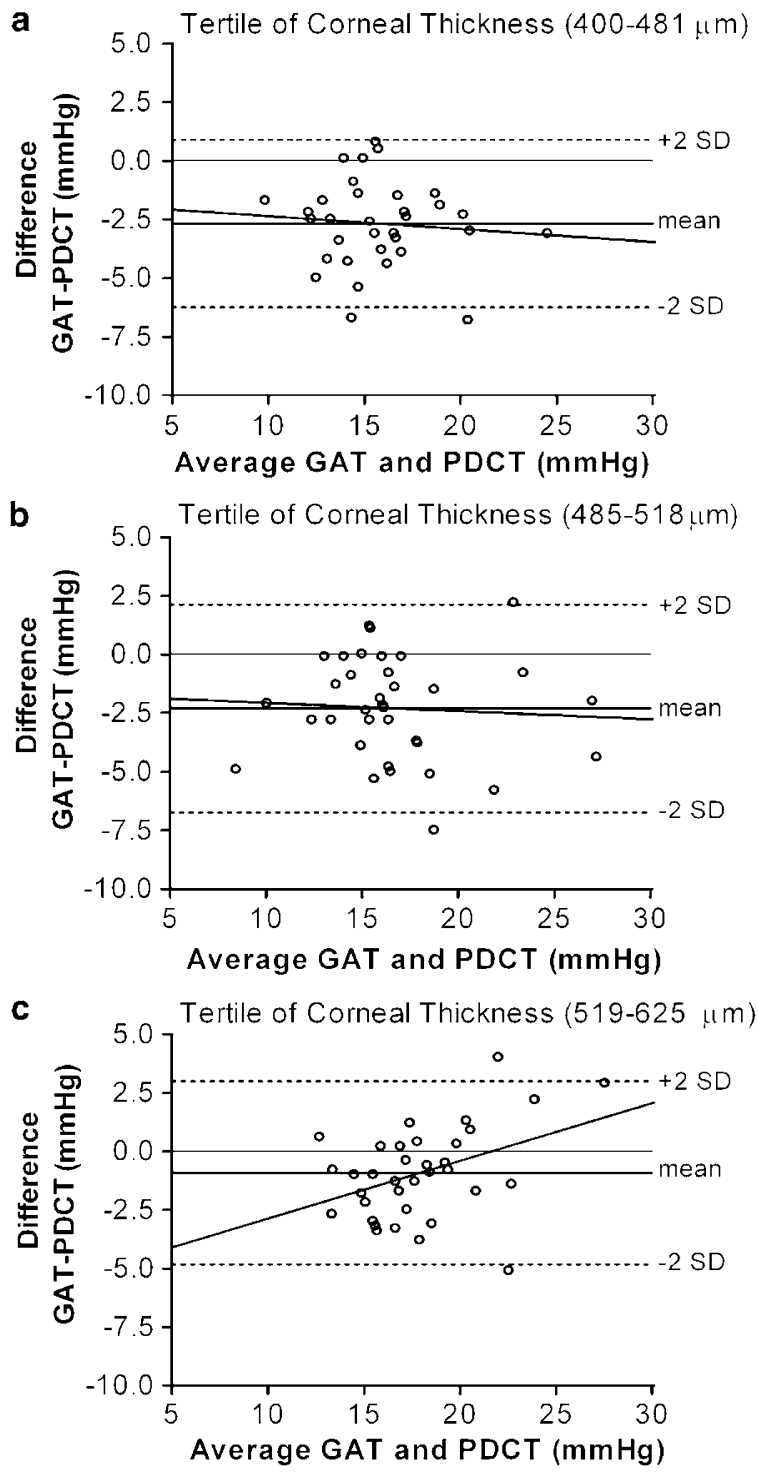

Figure 4 Bland and Altman plots for each tertile; (a) thin $=$ CCT from 400 to $481 \mu \mathrm{m}$, (b) intermediate $=$ CCT from 485 to $518 \mu \mathrm{m}$, and (c) thick =CCT from 519 to $625 \mu \mathrm{m}$. In the thin $\left(r^{2}=0.008\right)$ and intermediate $\left(r^{2}=0.004\right)$ groups, there was no significant mean difference between the two tonometers throughout the IOP range. However, in the thick group $\left(r^{2}=0.16\right)$, there was a trend for the GAT measurement to be greater than that of the PDCT when the IOP is above $20 \mathrm{mmHg}$.

Of the 106 patients, 36 (34\%) preferred the PDCT, 15 (14\%) preferred the GAT, and 55 (52\%) expressed no preference. Of 51 subjects who expressed a preference, three $(6 \%)$ did not provide reasons for their preference, while $13(25 \%)$ patients expressed more than one reason. Of those who preferred PDCT, their main reasons were: 'prefer yellow light over blue light' (28\%), 'PDCT appeared quicker to use' (16\%), 'easier to use' (16\%), 'sound effect assists cooperation during recording' (14\%), 
'no need for fluorescein' (14\%), and 'comfort' (12\%). Of those who preferred GAT, reasons were: 'comfort' $(41 \%)$, 'ease of operation' (24\%), 'speed of recording' (18\%), and 'preference of blue light over yellow light' (18\%).

\section{Discussion}

To date, the authors are aware of three published studies comparing IOP measurements with GAT and DCT in subjects undergoing myopic LASIK correction. ${ }^{21,24,25}$ Kaufmann et $a l^{21}$ compared IOP measurements using GAT and DCT preoperatively and 2 weeks after LASIK in 25 patients and highlighted a significant decrease in GAT-measured IOP postoperatively but no significant change in DCT measured IOP. However, this study was unmasked, limited by the small sample size, and no analysis of agreement between the instruments was attempted. Siganos et $a l^{24}$ compared PDCT with GAT in 118 eyes of 60 subjects and demonstrated an influence of CCT on GAT IOP measurements preoperatively, and post-LASIK, while demonstrating no effect of CCT on PDCT measurements. Despite a larger number of eyes, the inclusion of both eyes from each patient may introduce a systematic bias and the multivariate analysis of variance utilized has limited power to detect real, albeit smaller, differences with the PDCT as it focuses on group averages rather than analysing paired data sets. Most recently, Duba et $a l^{25}$ in a small sample using both eyes, evaluated 39 eyes of 20 subjects before and 3 months after LASIK and identified similar results.

In the current masked, prospective study, we compared GAT and PDCT in 106 eyes of 106 subjects with 'normal' corneas and assessed the influence of CCT on these instruments. It was noted that the PDCT tended to measure IOP $2.0 \pm 2.1 \mathrm{mmHg}$ higher than GAT, which is comparable with previous observations. ${ }^{21,25}$ This could be attributed to the calibration of the PDCT, which is based on a manometrically controlled pressure and not applanation. Although linear regression analysis showed good correlation between PDCT and GAT measurements, this represents an association only, conferring no information on agreement.

Using Bland and Altman analysis the current study highlights that in $95 \%$ of measurements, the PDCT IOP measurements fell within $\pm 4.2 \mathrm{mmHg}$ of the GAT IOP measurement (Figure 1). Interestingly, this is consistent with a manometric study on 73 human eyes, which identified the $95 \%$ limits of agreement between applanation and intracameral measurement to be $\pm 4.6 \mathrm{mmHg} .{ }^{26}$ This relatively poor agreement between the GAT and PDCT is clinically significant and might alter management decisions in many cases.

This study was powered after consideration of the clinical utility of the tests. Opinions vary as to the most appropriate cutoff for a clinically significant IOP change. The Early Manifest Glaucoma Trial reported that 'each higher (or lower) millimeter of mercury of IOP on followup was associated with an approximate $10 \%$ increased (or decreased) risk of progression' ${ }^{27}$ Other authors have defined a $1.5^{28}$ or $1.75 \mathrm{mmHg}^{29}$ difference as significant in their randomized clinical trial comparing the IOP lowering effects of topical prostaglandin analogues. Shin et $a l^{30}$ defined a CCT-associated IOP adjustment of $3.0 \mathrm{mmHg}$ or greater as 'outcomes significant' levels. Therefore, with that in mind, and with a cutoff level set at $2.0 \mathrm{mmHg}$, this study possesses the power to detect a difference of this magnitude, which would be clinically relevant.

The mean CCT in this study was $503.60 \pm 43.37 \mu \mathrm{m}$, which is slightly lower than that found in other studies based on ultrasonic pachymetry. ${ }^{5,6,10}$ All measurements were performed on the same calibrated ultrasound machine, by the same operator and within the same time period, hence it is unlikely that there is a random error bias in these measurements. However, although the ultrasound device was calibrated for the study, we cannot absolutely exclude a consistent slight undermeasurement that might be obtained by tissue compression. Interestingly, IOP measured by GAT correlated strongly with CCT $(r=0.37, P=0.0001)$ whereas, the relationship between IOP measured by PDCT and CCT approached but did not achieve significance $(r=0.17, P=0.073)$. This is consistent with previously published data. ${ }^{21,24,25}$ In addition, the difference between GAT- and PDCT-measured IOP showed similar correlation with CCT as the GAT $(r=0.37, P<0.0001)$ (Figure 3$)$. Linear regression showed the mean change in IOP measured by GAT was $2.8 \mathrm{mmHg}$ per $100 \mu \mathrm{m}$ change in CCT, also consistent with previously published observations. ${ }^{10,24}$ In contrast, IOP measured by PDCT only showed a mean change of $1 \mathrm{mmHg}$ per $100 \mu \mathrm{m}$ change in CCT. Consequently, use of GAT would lead to a difference of approximately $\pm 3 \mathrm{mmHg}$ based on the thickest $(625 \mu \mathrm{m})$ and the thinnest $(400 \mu \mathrm{m})$ cornea in this study, potentially influencing clinical management. Meanwhile, the smaller difference of $\pm 1 \mathrm{mmHg}$ when using the PDCT would be less likely to be considered clinically significant.

This relationship between CCT and IOP measurements was further supported by the data when stratified into tertiles by CCT. Subjects in the thickest tertile demonstrated a poorer agreement between the two instruments, compared to the intermediate or the thin groups. Furthermore, the GAT provided significantly higher IOP measurements in those with the thickest CCT $(P=0.003)$ while the IOP measured by PDCT was not affected by the difference in CCT $(P=0.37)$, suggesting this disparity between GAT and PDCT in the thickest 
tertile was due to the influence of CCT on GAT measurement. This was further supported by Bland and Altman analysis of the tertiles with a trend for the GAT measurement to be greater than that of the PDCT when the IOP is above $20 \mathrm{mmHg}$ in the thickest tertile (Figure $4 \mathrm{a}-\mathrm{c})$. This reflects, at least in part, the dependence of GAT measured IOP on CCT, which results in significant overestimation compared to the PDCT in patients with thicker corneas. Interestingly, a recent manometric study also showed greater disparity between GAT and the 'true IOP' ${ }^{31}$ However, in the mid-tertile one would have expected a lower variability between the IOP recordings of the two instruments if the difference was due solely to their different response to corneal thickness. Perhaps with larger sample size or with more eyes with higher IOPs this may have been demonstrated.

It was decided to analyse the data using Bland and Altman analysis of the tertiles because; (a) they showed the actual data across all corneal thicknesses; (b) they demonstrated that agreement between the two methodologies was different with increasing corneal thickness; (c) they summarized the magnitude of the differences with limits of agreement for three ranges of corneal thicknesses, which is likely to be more clinically meaningful than a simple function or equation relating error to a $1 \mu \mathrm{m}$ increase in corneal thickness. Obviously, there will be some regression dilution bias but with application of these methodologies in a clinical context, this is unavoidable.

All patients with glaucoma in this study were treated with topical glaucoma medications. This could theoretically distort the IOP/CCT relationships, although Korey et $a l^{32}$ studied 254 patients with normal IOP, untreated OHT, treated OHT and POAG and demonstrated no significant relationship between CCT and the use of ocular hypotensive medications. These findings were supported by Wang et al. ${ }^{33}$ Although Lass et $a l^{34}$ demonstrated a change in mean CCT with glaucoma medications; these were $1 \%$ or less at 12 months, which is within an acceptable range.

In normal clinical practice, unless there is corneal distortion, significant astigmatism or difficulty with obtaining an accurate measure, it is the author's general impression that clinicians generally take one IOP measurements per eye with GAT. Therefore, this was the methodology pursued in this study. In addition, although the average number of measurements per eye might theoretically improve the accuracy of IOP measurements, conversely, multiple measurements by applanation might artificially reduce the IOP. ${ }^{1}$

PDCT provides a noninvasive and rapid measurement of the OPA. This is potentially useful for the diagnosis of glaucoma ${ }^{35}$ and detection of cavernous sinus arteriovenous fistulae. ${ }^{36}$ In our study, the mean OPA was
$2.8 \pm 1.2 \mathrm{mmHg}$, a value slightly lower than that in another study utilizing the SmartLens dynamic observing tonometer, ${ }^{35}$ but in agreement with other studies based on a pneumotonometer linked to an ocular blood flow system. ${ }^{37,38}$ However, unlike Schwenn et al, ${ }^{35}$ we did not find a significant difference in OPA between subjects with and without glaucoma $(P=0.13)$.

However, the PDCT has several limitations. From a practical aspect, the requirement for sensor tip cover application with sterile air jet is cumbersome and time consuming. Although recently a new tip cover applicator has been introduced, which does not require the use of air jets. The disposable tip sensor cover also has an ongoing maintenance cost, which could limit its widespread use, although it has the benefit of reducing the risk of contamination. Specific requirements mentioned previously, need to be met to ensure that the IOP measurements are independent of corneal properties. Nonetheless, the majority of the population will fall within those criteria. The subjective survey showed that the majority of patients $(52 \%)$ did not have a preference for either tonometer. However, of those who expressed a preference, slightly more preferred PDCT $(34 \%)$ than GAT $(14 \%)$. The main reason cited was the preference for yellow over blue light illumination. Several patients also commented that the audio cue allowed better cooperation as they could concentrate on keeping their eye open during the actual recording.

This study demonstrates correlation of GAT IOP measurements with CCT while PDCT IOP measurements were less dependent on CCT in a group of patients with normal corneas. Although the agreement was poor between PDCT and GAT IOP measurements, this may reflect inherent inaccuracies of either the current gold standard (GAT) or that of PDCT. These initial data supports a potential clinical role for the PDCT, particularly for subjects with CCT outside of the normal range. However, the analysis of its precision should be a subject of further studies.

\section{Acknowledgements}

The study was partially supported by unrestricted research funding from: the Alcon glaucoma and neuroophthalmology fellowship fund, and the Sir William and Lady Stevenson Trust. We thank Designs for Vision for the unrestricted loan of the Pascal dynamic contour tonometer used in this study.

\section{Statement}

The authors have no proprietary or commercial interest in the equipment or studies presented in this paper. 


\section{Ethics approval}

This study was approved by the Auckland Ethics Committee, Auckland, New Zealand.

\section{References}

1 Whitacre MM, Stein R. Sources of error with use of Goldmann-type tonometers. Surv Ophthalmol 1993; 38(1): 1-30.

2 Goldmann V, Schmidt T. Uber Applanationstonometrie. Ophthalmologica 1957; 134: 221-242.

3 Hansen FK. A clinical study of the normal human central corneal thickness. Acta Ophthalmologica 1971; 49(1): 82-99.

4 Herman DC, Hodge DO, Bourne WM. Increased corneal thickness in patients with ocular hypertension. Arch Ophthalmol 2001; 119(3): 334-336.

5 Wolfs RC, Klaver CC, Vingerling JR, Grobbee DE, Hofman A, de Jong PT. Distribution of central corneal thickness and its association with intraocular pressure: the Rotterdam Study. Am J Ophthalmol 1997; 123(6): 767-772.

6 Copt RP, Thomas R, Mermoud A. Corneal thickness in ocular hypertension, primary open-angle glaucoma, and normal tension glaucoma. Arch Ophthalmol 1999; 117(1): $14-16$.

7 Doughty MJ, Zaman ML. Human corneal thickness and its impact on intraocular pressure measures: a review and meta-analysis approach. Surv Ophthalmol 2000; 44(5): 367-408.

8 Ehlers N, Bramsen T, Sperling S. Applanation tonometry and central corneal thickness. Acta Ophthalmologica 1975; 53(1): 34-43.

9 Whitacre MM, Stein RA, Hassanein K. The effect of corneal thickness on applanation tonometry. Am J Ophthalmol 1993; 115(5): 592-596.

10 Bron AM, Creuzot-Garcher C, Goudeau-Boutillon S, d'Athis P. Falsely elevated intraocular pressure due to increased central corneal thickness. Graefes Arch Clin Exp Ophthalmol 1999; 237(3): 220-224.

11 Herndon LW, Choudhri SA, Cox T, Damji KF, Shields MB, Allingham RR. Central corneal thickness in normal, glaucomatous, and ocular hypertensive eyes. Arch Ophthalmol 1997; 115(9): 1137-1141.

12 Gordon MO, Beiser JA, Brandt JD, Heuer DK, Higginbotham EJ, Johnson CA et al. The Ocular Hypertension Treatment Study: baseline factors that predict the onset of primary open-angle glaucoma. Arch Ophthalmol 2002; 120(6): 714-720.

13 Kass MA, Heuer DK, Higginbotham EJ, Johnson CA, Keltner JL, Miller JP et al. The Ocular Hypertension Treatment Study: a randomized trial determines that topical ocular hypotensive medication delays or prevents the onset of primary open-angle glaucoma. Arch Ophthalmol 2002; 120(6): 701-713.

14 Phelan PS, McGhee CN, Bryce IG. Excimer laser PRK and corticosteroid induced IOP elevation: the tip of an emerging iceberg? Br J Ophthalmol 1994; 78(10): 802-803.

15 Zadok D, Tran DB, Twa M, Carpenter M, Schanzlin DJ. Pneumotonometry versus Goldmann tonometry after laser in situ keratomileusis for myopia. J Cataract Refract Surg 1999; 25(10): 1344-1348.

16 Mardelli PG, Piebenga LW, Whitacre MM, Siegmund KD. The effect of excimer laser photorefractive keratectomy on intraocular pressure measurements using the Goldmann applanation tonometer. Ophthalmol 1997; 104(6): 945-948.

17 Chatterjee A, Shah S, Bessant DA, Naroo SA, Doyle SJ Reduction in intraocular pressure after excimer laser photorefractive keratectomy. Correlation with pretreatment myopia. Ophthalmology 1997; 104(3): 355-359.

18 Shaikh NM, Shaikh S, Singh K, Manche E. Progression to end-stage glaucoma after laser in situ keratomileusis. J Cataract Refract Surg 2002; 28(2): 356-359.

19 Hamilton DR, Manche EE, Rich LF, Maloney RK. Steroidinduced glaucoma after laser in situ keratomileusis associated with interface fluid. Ophthalmology 2002; 109(4): 659-665.

20 Mitchell P, Hourihan F, Sandbach J, Wang JJ. The relationship between glaucoma and myopia: the Blue Mountains Eye Study. Ophthalmol 1999; 106(10): 2010-2015.

21 Kaufmann C, Bachmann LM, Thiel MA. Intraocular pressure measurements using dynamic contour tonometry after laser in situ keratomileusis. Invest Ophthalmol Vis Sci 2003; 44(9): 3790-3794.

22 SMT Swiss Microtechnology AG. Pascal Dynamic Contour Tonometer Operating Manual, version 1.4, Swiss Microtechnology: Switzerland, 2004, pp 30-32.

23 Bland J, Altman D. Statistical methods for assessing agreement between two methods of clinical measurement. Lancet 1986; 1: 307-310.

24 Siganos DS, Papastergiou GI, Moedas C. Assessment of the Pascal dynamic contour tonometer in monitoring intraocular pressure in unoperated eyes and eyes after LASIK. J Cataract Refract Surg 2004; 30(4): 746-751.

25 Duba I, Wirthlin AC. Dynamic contour tonometry for post-LASIK intraocular pressure measurements. Klin Monatsbl fur Augenheilkd 2004; 221(5): 347-350.

26 Feltgen N, Leifert D, Funk J. Correlation between central corneal thickness, applanation tonometry, and direct intracameral IOP readings. Br J Ophthalmol 2001; 85(1): 85-87.

27 Leske MC, Heijl A, Hussein M, Bengtsson B, Hyman L, Komaroff $\mathrm{E}$ et al. Factors for glaucoma progression and the effect of treatment. Arch Ophthalmol 2003; 121: 48-56.

28 Parrish RK, Palmberg P, Sheu WP, XLT Study Group. A comparison of latanoprost, bimatoprost, and travoprost in patients with elevated intraocular pressure: a 12 week, randomized, masked-evaluator multicenter study. $A m \mathrm{~J}$ Ophthalmol 2003; 135: 688-703.

29 Noecker RS, Dirks MS, Choplin NT, Bernstein P, Batoosingh $\mathrm{AL}$, Whitcup SM et al. A six-month randomized clinical trial comparing the intraocular pressure-lowering efficacy of bimatoprost and latanoprost in patients with ocular hypertension or glaucoma. Am J Ophthalmol 2003; 135: 55-63.

30 Shih CY, Graff ZJS, Trokel SL, Tsai JC. Clinical significance of central corneal thickness in the management of glaucoma. Arch Ophthalmol 2004; 122(9): 1270-1275.

31 Foster PJ, Wong JS, Wong E, Chen FG, Machin D, Chew PT. Accuracy of clinical estimates of intraocular pressure in Chinese eyes. Ophthalmology 2000; 107(10): 1816-1821.

32 Korey M, Gieser D, Kass MA, Waltman SR, Gordon M, Becker B. Central corneal endothelial cell density and central corneal thickness in ocular hypertension and primary open-angle glaucoma. Am J Ophthalmol 1982; 94(5): 610-616. 
33 Wang TH, Huang JY, Hung PT, Shieh JW, Chen YF. Ocular hypotensive effect and safety of brinzolamide ophthalmic solution in open angle glaucoma patients. J Formos Med Assoc 2004; 103(5): 369-373.

34 Lass JH, Eriksson GL, Osterling L, Simpson CV, Latanoprost Corneal Effects Study Group. Comparison of the corneal effects of latanoprost, fixed combination latanoprosttimolol, and timolol: a double-masked, randomized, oneyear study. Ophthalmology 2001; 108(2): 264-271.

35 Schwenn O, Troost R, Vogel A, Grus F, Beck S, Pfeiffer N. Ocular pulse amplitude in patients with open angle glaucoma, normal tension glaucoma, and ocular hypertension. Br J Ophthalmol 2002; 86(9): 981-984.
36 Kaufmann C, Thiel MA, Valavanis A, Landau K, Duba I, Wirthlin AC. Normalisation of ocular pulse amplitude after embolisation of dural cavernous sinus arteriovenous fistula. Klin Monatsbl fur Augenheilkd 2004; 221(5): 431-434.

37 Kerr J, Nelson P, O'Brien C. A comparison of ocular blood flow in untreated primary open-angle glaucoma and ocular hypertension. Am J Ophthalmol 1998; 126(1): 42-51.

38 Georgopoulos GT, Diestelhorst M, Fisher R, Ruokonen P, Krieglstein GK. The short-term effect of latanoprost on intraocular pressure and pulsatile ocular blood flow. Acta Ophthalmol Scand 2002; 80(1): 54-58. 